\title{
Impaired Surface Expression of HLA-DR, TLR2, TLR4, and TLR9 in Ex Vivo-In Vitro Stimulated Monocytes from Severely Injured Trauma Patients
}

\author{
David Heftrig, ${ }^{1}$ Ramona Sturm, ${ }^{1}$ Elsie Oppermann, ${ }^{2}$ Kerstin Kontradowitz, ${ }^{1}$ Katrin Jurida, \\ Lukas Schimunek, ${ }^{1}$ Mathias Woschek, ${ }^{1}$ Ingo Marzi, ${ }^{1}$ and Borna Relja ${ }^{1}$ \\ ${ }^{1}$ Department of Trauma, Hand and Reconstructive Surgery, Goethe University, Frankfurt, Germany \\ ${ }^{2}$ Department of General and Visceral Surgery, Goethe University, Frankfurt, Germany \\ Correspondence should be addressed to Borna Relja; info@bornarelja.com
}

Received 30 August 2016; Revised 8 November 2016; Accepted 27 November 2016; Published 1 February 2017

Academic Editor: Fumio Tsuji

Copyright (C) 2017 David Heftrig et al. This is an open access article distributed under the Creative Commons Attribution License, which permits unrestricted use, distribution, and reproduction in any medium, provided the original work is properly cited.

\begin{abstract}
Objective. Trauma patients (TP) frequently develop an imbalanced immune response that often causes infectious postinjury complications. Monocytes show a diminished capability of both producing proinflammatory cytokines and antigen presentation after trauma. TLR2, TLR4, and TLR9 recognize pathogens and subsequently activate monocytes. While there are conflictive data about TLR2 and TLR4 expression after trauma, no studies about the expression of TLR2, TLR4, TLR9, and HLA-DR on monocytes from TP after their secondary ex vivo-in vitro "hit" have been reported. Methods/Results. Ex vivo-in vitro lipopolysaccharide- (LPS) stimulated blood from TP showed diminished interleukin- (IL-) $1 \beta$-release in TP for five postinjury days compared to healthy volunteers (HV). The recovery was observed at day 5. In parallel, monocytes from TP showed an impaired capability of TLR2, TLR4, and TLR9 expression after secondary stimulation compared to HV, while the measurement of unstimulated samples showed significant reduction of TLR4 and TLR9 at ED. Furthermore, HLA-DR decreased after trauma and was even more profound by stimulation of monocytes. Ratio of monocytes to leukocytes was significantly increased at days 6 and 7 after trauma compared to HV. Conclusion. Impaired expression of TLRs and HLA-DR in acute inflammatory conditions may be responsible for the welldescribed monocyte paralysis after severe trauma.
\end{abstract}

\section{Introduction}

Despite recent significant improvements with regard to the treatment of severely injured trauma patients (TP), clinical complications, which develop due to increased susceptibility to opportunistic infections, are often $[1,2]$. Trauma patients constitute a highly heterogeneous cohort of patients regarding their immune response. However, sepsis and multiorgan failures (MOF) are still predominant causes of late mortality after trauma $[2,3]$.

In response to injury, the innate and adaptive immune system is activated. The release of damage-associated molecular patterns (DAMPs) caused by tissue damage induces a systemic inflammatory response that attempts to neutralize the pathogen microorganisms and initiate the tissue repair mechanisms [4]. This process is defined as the systemic inflammatory response syndrome (SIRS) [5]. In parallel, trauma causes a counterbalancing immune response, so-called compensatory anti-inflammatory response syndrome (CARS) $[6,7]$. The simultaneous development of SIRS and CARS results in a mixed antagonist response syndrome (MARS) [7]. Therefore, intensive care patients who initially survive the trauma impact may still undergo a persistent inflammation, immunosuppression, and catabolism syndrome (PICS) [8].

As critical regulators of the immune system, human monocytes exert a decreased capability of releasing proinflammatory cytokines such as tumor necrosis factor-alpha (TNF-alpha) and interleukin-1-beta (IL-1 $\beta$ ) after a secondary ex vivo-in vitro exposure to endotoxin after trauma [9-11]. This has been illustrated on postinjury days 2, 5, and 10 [12]. On the other hand, other studies described a monocytosis after trauma and surgical sepsis $[13,14]$. Interestingly, expression of the major histocompatibility complex 2 (MHC 2) and 
activator of the T-cell receptor human leukocyte antigenDR (HLA-DR) decreased on monocyte's surface (mHLADR) after trauma $[15,16]$. Persisting low levels of mHLA-DR have been associated with major sepsis development, while patients with uneventful recovery reached normal levels of mHLA-DR after trauma $[15,17]$. The decreased immune response to tissue injury carries the potential for immunological dysfunctions, which often cause infections and/or multiorgan disorders in trauma patients [18].

DAMPs arise from tissue damage and are recognized by leukocytes via their pathogen recognition receptors (PRRs) [19]. The toll-like receptors (TLRs, in mammalian 13 different types) are specific subtypes of PRRs $[20,21]$. TLRs play an important role in the intracellular signalling and subsequent induction of the innate immunity [22]. TLR2 is activated by different ligands such as peptidoglycan and lipoteichoic acid deriving from Gram-positive microorganisms [23, 24]. TLR4 recognizes endotoxins of Gram-negative bacteria (e.g., lipopolysaccharide (LPS)) [25]. Together with CD14 and the LPS-binding protein (LBP), TLR4 activates monocytes and subsequently increases both transcription and the release of proinflammatory cytokines [26, 27]. By recognizing of bacterial DNA with high amounts of unmethylated CpG dinucleotides TLR9 activates the immune response [28, 29]. In several studies, TLR9 is described as an intracellular receptor; however, there are reports describing its surface expression as well [30-33]. Compared to control subjects, trauma patients exert an impaired capability to produce proinflammatory cytokines after CpG-oligonucleotide stimulation [34]. Different expression patterns of TLRs on circulating monocytes have been reported after trauma. Pérez-Bárcena et al. have postulated higher expressions of TLR2 and TLR4 [35], while others detected reduced TLR4 expression, with unchanged TLR2 [34], reduced TLR2 expression with unchanged TLR4 expression [36], and reduced expression of both TLR2 and TLR4 in TP [37]. Taken together, these studies do not provide an explanation for the diminished cytokine release and impaired activity of monocytes after trauma. Furthermore, the expression analysis of TLRs after a secondary ex vivo-in vitro stimulation as described in this study has never been performed before in trauma patients.

Here, the expression and coexpression of TLR2, TLR4, TLR9, and of HLA-DR were measured on circulating CD14 positive monocytes after severe trauma. In order to simulate secondary acute inflammatory conditions after trauma, the samples were stimulated with a leukocyte activation cocktail (LAC) and the (co-)expression of these receptors was evaluated. In addition, a daily analysis of the IL- $1 \beta$ releasing capability of patient's monocytes after their LPS stimulation was conducted until posttrauma day 10 .

\section{Patients and Methods}

2.1. Ethics. The study was performed in the University Hospital Frankfurt, Goethe University, Germany, with the institutional ethical committee approval (312/10) in accordance with the Declaration of Helsinki and following STROBE guidelines [38]. All patients or their legally authorized representative as well as included healthy volunteers signed the written informed consent form.

2.2. Patients. In our prospective clinical experimental trial, 29 severely injured trauma patients with a history of acute blunt or penetrating trauma and an injury severity score (ISS) $\geq 16$ were enrolled. Exclusion criteria were being younger than 18 or older than 80 years of age, severe burn injury, acute myocardial stroke, cancer or chemotherapy, immunosuppressive drug therapy, HIV, infectious hepatitis, acute CMV infection, and/or thromboembolic events. Upon arrival at the emergency department (ED), vital signs were measured and the ISS was calculated according to the abbreviated injury scale (AIS) as of 2008 [39, 40]. The control group consisted of 14 healthy volunteers (HV).

2.3. Blood Sampling. Blood samples were withdrawn in ethylenediaminetetraacetic acid (EDTA) tubes (Sarstedt, Nürmbrecht, Germany) directly after admission to the ED and daily until day 10 after trauma. The samples were kept either at room temperature for functional assays or on ice for flow cytometric analysis. The subsequent blood samples taken daily from $\mathrm{TP}$ as well as blood samples from $\mathrm{HV}$ were obtained between 7 and 11 a.m.

2.4. Ex Vivo-In Vitro Whole Blood Stimulation for Cytokine Production Assay. Blood samples $(50 \mu \mathrm{l})$ were diluted in $450 \mu \mathrm{l}$ RPMI 1640 (Seromed, Berlin, Germany; polypropylene tube, BD Bioscience, Franklin Lakes, NJ, USA) supplemented with $10 \%$ heat-inactivated fetal calf serum (FCS), $100 \mathrm{IU} / \mathrm{mL}$ penicillin, and $100 \mu \mathrm{g} / \mathrm{mL}$ streptomycin (Gibco, Karlsruhe, Germany) and $20 \mathrm{mM}$ HEPES buffer (Sigma, Deisenhofen, Germany). The samples were stimulated with LPS (5 $\mu \mathrm{g} / \mathrm{ml}$, E. coli 0111:B4, SIGMA-Aldrich) and incubated at $37^{\circ} \mathrm{C}$ and $5 \% \mathrm{CO}_{2}$. Twenty-four h later, the samples were centrifuged at $2100 \mathrm{~g}$ for 15 minutes, and the supernatant was collected and stored at $-80^{\circ} \mathrm{C}$ until assay. To address unspecific stimulation, corresponding blood samples were incubated as described above without LPS stimulation.

The samples were assayed for IL- $1 \beta$ using the enzymelinked immunosorbent assay (ELISA) technique (Quantikine $^{\circledR}$, Human IL-1 $\beta /$ IL-1F2 Immunoassay ELISA, R\&D Systems) according to the manufacturer's instructions.

2.5. Ex Vivo-In Vitro Whole Blood Stimulation for Cell Surface Receptor Analysis. Blood samples $(100 \mu \mathrm{l})$ were diluted in $395 \mu \mathrm{l}$ RPMI 1640, and $5 \mu \mathrm{l}$ of the leukocyte activation cocktail (LAC, containing the phorbol ester, phorbol 12-myristate 13acetate (PMA), a calcium ionophore (Ionomycin), and the protein transport inhibitor BD GolgiPlug ${ }^{\mathrm{TM}}$ (Brefeldin A), BD Pharmingen ${ }^{\mathrm{TM}}$ ) were added. The samples were incubated for $5 \mathrm{~h}$ at $37^{\circ} \mathrm{C}$ under $5 \% \mathrm{CO}_{2}$ and stained for flow cytometric analysis as described below. Unstimulated corresponding samples were evaluated as controls.

2.6. Measurement of Cell Surface Receptor Expression by Flow Cytometry. Blood samples $(100 \mu \mathrm{l})$ were transferred into polystyrene FACS tubes (BD Pharmingen) and incubated with mouse anti-human CD14 V500 (Clone M5E2, BD 


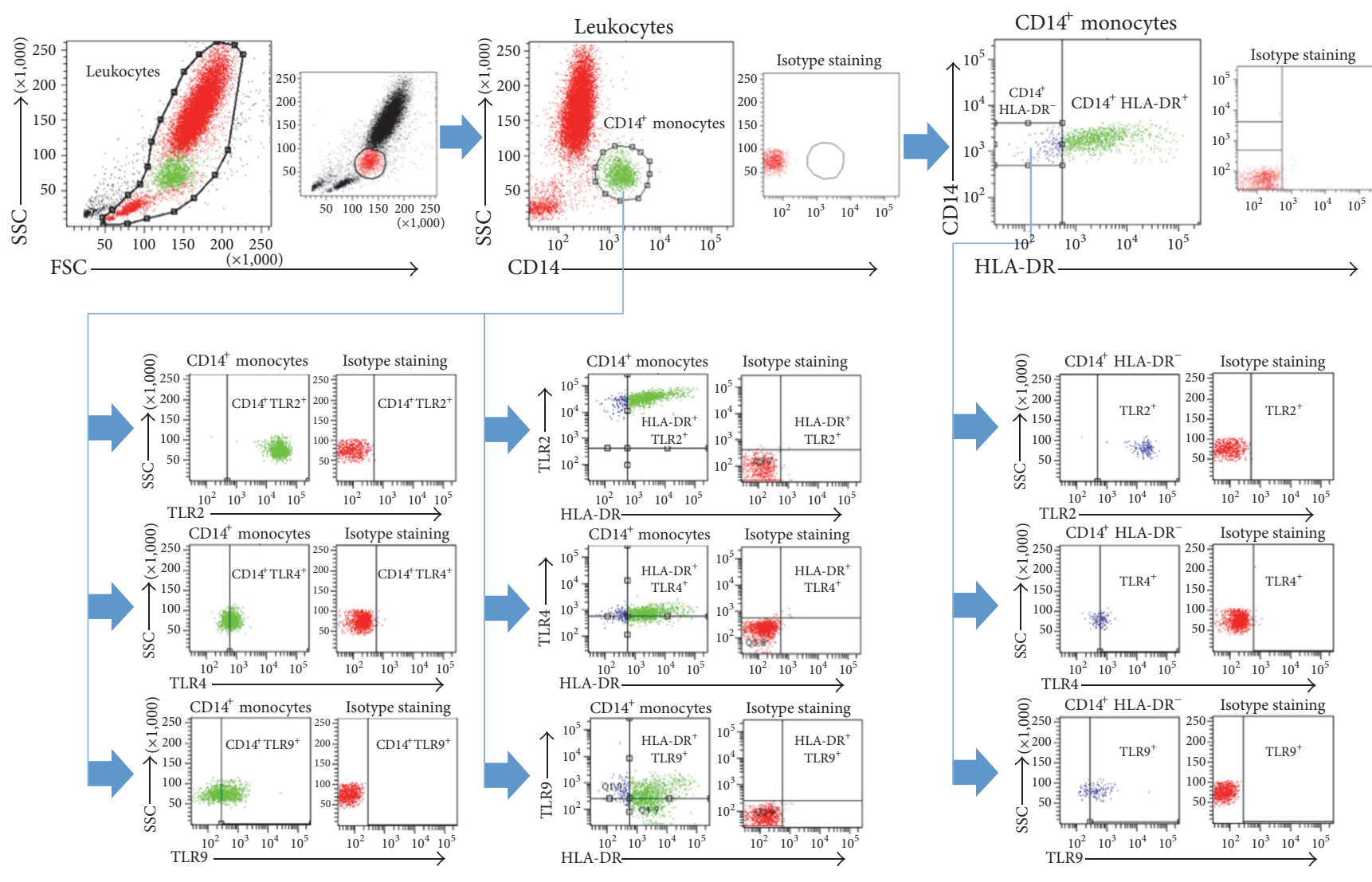

FIGURE 1: Gating strategy for the flow cytometric analysis and evaluation.

Bioscience, San Jose, CA), mouse anti-human TLR2 PECy7 (Clone T2.5, eBioscience, San Diego, CA), mouse antihuman TLR4 FITC (Clone HTA125, IMGENEX, San Diego, CA), mouse anti-human TLR9 Alexa Flour ${ }^{\circledR} 647$ (Clone 26C593.2, IMGENEX, San Diego, CA), and mouse antihuman HLA-DR PerCP-Cy5.5 conjugated (Clone L243, BD Bioscience, San Jose, CA) antibodies. After 30 minutes at room temperature, $3 \mathrm{ml}$ of FACS lysing solution (FACS Lysing Solution, BD Pharmingen) was added for additional 10 minutes. Subsequently, the samples were centrifuged at $400 \mathrm{~g}$ for 5 minutes and washed twice with $4 \mathrm{ml}$ phosphatebuffered saline (PBS) supplemented with $0.5 \%$ bovine serum albumin (FACS buffer). Immediately after the supernatants were removed, the cells were diluted in $300 \mu$ l FACS buffer and were then subjected to flow cytometry with a $B D$ FACS Canto $2^{\mathrm{TM}}$ using FACD DIVA ${ }^{\mathrm{TM}}$ software (BD). The monocyte population was defined by gating $\mathrm{CD}_{1}{ }^{+}$cells in the corresponding forward and side scatter scan. From each sample, a minimum of 20.000 monocytes was measured. The number of totally gated cells for each was calculated as absolute cell number in percentage relative to the ratio of the indicated cell populations in representative figures. The gating strategy is shown in Figure 1.

2.7. Statistical Analysis. GraphPad Prism 6.0 software (GraphPad Software Inc., San Diego, CA) was used to perform the statistical analysis. Data are given as mean \pm standard error of the mean (SEM) or as absolute cell numbers calculated in percent. Student's $t$-test with Welch correction and one-way analysis of variance (ANOVA) with a Dunn post hoc test were used for comparison among all different groups. A $p$ value below 0.05 was considered statistically significant.

\section{Results}

3.1. Study Population. 29 patients with major trauma (TP) and $14 \mathrm{HV}$ were enrolled in this study. The majority of the study subjects were male (TP: 76\% versus HV: 64\%). The mean age of TP was $46 \pm 3$ versus $37 \pm 6$ in HV. All patients were substantially injured (ISS: $28 \pm 2$ ). The mean stay in the intensive care unit (ICU) was $10 \pm 2.0$ days. The in-hospital stay duration was $20 \pm 4$ days. In moderate contrast to our previous studies [11, 41, 42], patients in this study represent the cohort of major trauma patients.

3.2. Time Course of the LPS Response in Whole Blood from Trauma Patients. Directly after admission, the IL- $1 \beta$ release was significantly decreased until day seven in TP compared to HV after LPS stimulation (ED: $663.0 \pm 102.4$ versus $1490.0 \pm$ $340.5 \mathrm{pg} / \mathrm{mL} ; p<0.05$, Figure 2$)$. A trend to a continuous IL$1 \beta$ secretion recovery in cells from TP was observed at day 5 after trauma. Even after 7 postinjury days, the IL-1 $\beta$ release was not recovered completely. Neither the nonstimulated samples of HV nor the nonstimulated samples of TP have shown any significant alterations in IL-1 $\beta$ release. 


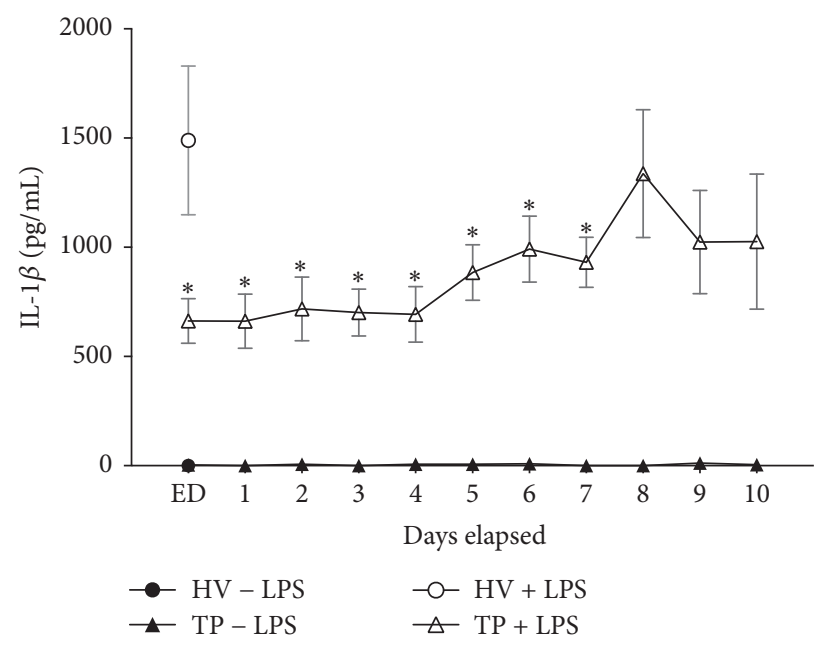

FIGURE 2: Major trauma leads to reduced IL-1 $\beta$ release from whole blood after LPS stimulation. Whole blood from healthy volunteers (HV, $n=14$ ) or major trauma patients (TP, $n=29$ ) was stimulated with LPS. Supernatants were collected after $24 \mathrm{~h}$ for measurements of IL-1 $\beta$ by ELISA. A 10-day time course after admission (emergency department, ED-10) was made. To address unspecific stimulation, corresponding blood samples were incubated as described above without LPS stimulation. Data are shown as mean \pm SEM. ${ }^{*} p<0.05$ versus LPS-stimulated HV.

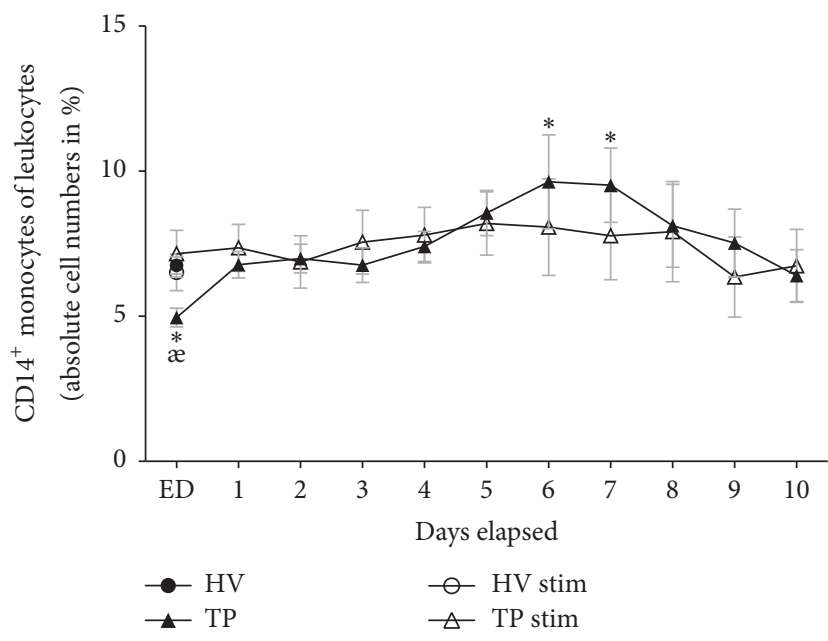

(a)

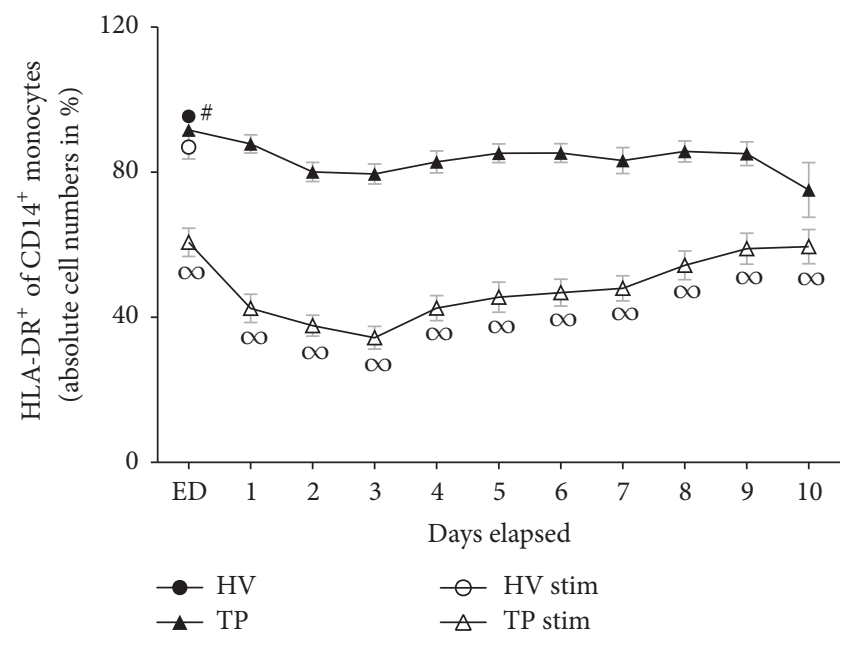

(b)

FIGURE 3: Whole blood from healthy volunteers ( $\mathrm{HV}, n=14)$ or major trauma patients (TP, $n=29)$ was analyzed by flow cytometry over a 10-day time course after admission (emergency department, ED-10). Monocytes were detected using anti-human CD14 in the corresponding sideward and forward scatter. Unstimulated (black symbols) and stimulated (clear symbols) measurements were made. For stimulation (stim), whole blood was incubated with leukocyte activation cocktail for $5 \mathrm{~h}$ with subsequent analyzing procedure as in unstimulated samples. Data are shown as mean \pm SEM. (a) CD14 ${ }^{+}$monocytes to leukocytes ratio, (b) HLA-DR expression in CD14 ${ }^{+}$monocytes. ${ }^{*} p<0.05$ versus unstimulated HV; ${ }^{x} p<0.05 \mathrm{TP}$ versus corresponding TP stim; ${ }^{\#} p<0.05 \mathrm{HV}$ ctrl versus all; ${ }^{\infty} p<0.05 \mathrm{TP}$ stim versus corresponding unstimulated TP and HV stim.

3.3. Ratio of $\mathrm{CD}^{+} 4^{+}$Monocytes to Leukocytes. TP had a significantly lower absolute cell numbers of CD14 ${ }^{+}$monocytes in total leukocyte population at ED compared to HV $(4.95 \pm 0.32$ versus $6.76 \pm 0.30$ absolute cell number in $\%, p<0.05$, Figure 3(a)). Thus, on day 5 , an increase in $\mathrm{CD} 14^{+}$monocytes was observed, with a significant maximum height on days 6 and 7 compared to HV (day 5: $8.56 \pm 0.78$; day 6: $9.64 \pm 1.62$; day $7: 9.51 \pm 1.28$ versus $6.76 \pm 0.30$ absolute cell number in $\%, p<0.05$, Figure $3(\mathrm{a}))$. At the end of observation period, the ratio of CD14 $4^{+}$monocytes to leukocytes in TP was comparable with HV. Stimulating whole blood samples with
LAC did not markedly change the ratio of $\mathrm{CD} 14^{+}$monocytes to leucocytes in HV and TP (HV: $6.51 \pm 0.63$, Figure 3(a)).

3.4. Surface Expression of mHLA-DR on CD14 ${ }^{+}$Monocytes. During the whole observational period, the ratio of HLA$\mathrm{DR}^{+}$cells to total CD14 $4^{+}$monocytes remained significantly lower compared to HV ( $p<0.05$, Figure 3(b)). After the ex vivo-in vitro stimulation with LAC, HLA-DR expression on $\mathrm{CD}_{1}{ }^{+}$monocytes from $\mathrm{HV}$ decreased significantly compared to unstimulated cells from HV $(86.96 \pm 3.28$ versus $95.43 \pm 1.35$ absolute cell number in $\%, p<0.05$, Figure $3(\mathrm{~b}))$. 
Stimulation of samples from TP reduced strongly the HLADR expression in $\mathrm{CD}_{1}{ }^{+}$monocytes compared to both unstimulated samples from TP and stimulated samples from HV (ED: $60.68 \pm 3.92$ versus $91.67 \pm 1.47$ and $86.96 \pm 3.28$ absolute cell number in \%, resp., $p<0.05$, Figure 3(b)). The loss of surface HLA-DR expression after LAC stimulation in $\mathrm{HV}$ resulted in an $8.9 \%$ decrease, whereas the loss of surface HLA-DR expression in TP after LAC stimulation was significantly higher, that is, $33.8 \%$ in samples from ED $(p<$ 0.05 , Figure 3(b)).

3.5. Surface Expression of TLR2 on CD14 ${ }^{+}$Monocytes. $99.4 \%$ of all $\mathrm{CD}_{14}{ }^{+}$monocytes from HV expressed TLR2. After trauma, that value did not change significantly (Figure 4(a)). LAC stimulation in samples from $\mathrm{HV}$ resulted in a significant decrease of TLR2 expression (97.2\%, $p<0.05$, Figure 4(a)). The TLR2 expression in $\mathrm{CD}_{1} 4^{+}$monocytes from TP was significantly decreased during the complete time course compared to unstimulated cells from TP as well compared to LAC-stimulated samples from HV (TP ED: $94.7 \pm 0.8$ versus $99.0 \pm 0.2 \%$ and $97.2 \pm 0.8 \%$, resp., $p<0.05$, Figure $4(\mathrm{a}))$.

3.6. Surface Expression of TLR4 on CD14 ${ }^{+}$Monocytes. $58.6 \%$ of all CD $14^{+}$monocytes out of the HV group expressed TLR4. Directly after admission, the value was significantly lower in TP (50.2\%, Figure 4(b)). During the observational period, it rose markedly beginning at day $1(60.6 \pm 4.1 \%)$ and showing no further significant changes during the posttraumatic time course compared to HV. LAC stimulation increased significantly the TLR4 expression in HV to $81.7 \%(p<0.05$, Figure 4(b)). The TLR4 expression in TP was significantly enhanced after LAC stimulation compared to unstimulated samples from TP (TP ED: $57.7 \pm 3.5$ versus $50.2 \pm$ $2.9 \%$, Figure 4(b)). Despite the continuously increased TLR4 expression on $\mathrm{CD}_{1} 4^{+}$monocytes in LAC-stimulated samples obtained from TP, the expression levels were still significantly lowered during the complete observational period compared to stimulated HV samples (Figure 4(b)).

3.7. Surface Expression of TLR9 on CD14 ${ }^{+}$Monocytes. $34.4 \%$ of all CD14 ${ }^{+}$monocytes from HV expressed TLR9. Upon arrival to the ED, the value was significantly decreased to 24.0\% ( $p<0.05$, Figure 4(c)). Nonetheless, the expression of TLR9 was comparable to the levels of HV after day 1. LAC stimulation increased significantly the TLR9 expression in $\mathrm{HV}$ and in TP during the whole observational period compared to unstimulated samples (HV: $89.9 \%$ versus $34.4 \%$, TP ED $77.6 \pm 3.8$ versus $24.0 \pm 1.6 \%, p<0.05$, Figure $4(\mathrm{c})$ ). However, the levels of TLR9 expression after LAC stimulation were significantly lower until day 8 after trauma compared to HV $(p<0.05$, Figure 4(c)). After day 8, TLR9 expression after LAC stimulation was comparable to stimulated $\mathrm{HV}$ samples.

\subsection{Surface Expression of TLR2, TLR4, and TLR9 on HLA-DR ${ }^{+}$or HLA-DR ${ }^{-} \mathrm{CD} 14^{+}$Monocytes}

3.8.1. Surface Expression of TLR2 on HLA-DR ${ }^{-}$and HLA-DR ${ }^{+}$ CD14 ${ }^{+}$Monocytes. $85.4 \%$ of all CD $14^{+} \mathrm{HLA}^{-D R^{-}}$monocytes from $\mathrm{HV}$ expressed TLR2. Upon arrival to the ED, $90.5 \%$ of $\mathrm{CD}_{14}{ }^{+} \mathrm{HLA}^{-D R^{-}}$monocytes from TP expressed TLR2, a value that was increased compared to $\mathrm{HV}$, but this difference was not significant. During the subsequent whole posttraumatic observation period, the TLR2 expression was significantly increased compared to HV. LAC stimulation increased the TLR2 expression of HV to 90.7\%; however, this increase was not significant (Figure 4(d)). Starting from day one until day six, LAC stimulation of samples from TP significantly increased the TLR2 expression compared to stimulated HV controls $(p<0.05$, Figure $4(\mathrm{~d}))$.

In contrast, the TLR2 expression on HLA-DR ${ }^{+} \mathrm{CD} 14^{+}$ monocytes showed no significant changes between $\mathrm{HV}$ and TP (HV: 99.7\%, data not shown). Thus, LAC stimulation significantly decreased the TLR2 expression in TP during the complete study period in contrast to stimulated HV controls (data not shown).

3.8.2. Surface Expression of TLR4 on HLA-DR ${ }^{-}$and $H L A-D R^{+}$ CD14 ${ }^{+}$Monocytes. $71.9 \%$ of all HLA-DR ${ }^{-} \mathrm{CD}_{14}{ }^{+}$monocytes from HV expressed TLR4 (Figure 4(e)). Starting at day 1 until day 10, the TLR4 expression in HLA-DR ${ }^{-} \mathrm{CD}_{14}{ }^{+}$monocytes was significantly decreased in TP. LAC stimulation increased significantly the TLR4 expression to 84.0 in $\mathrm{HV}(p<0.05$, Figure $4(\mathrm{e}))$. During the whole posttraumatic observational period, the TLR4 expression after stimulation was significantly decreased in TP compared to stimulated HV.

The TLR4 expression in HLA-DR ${ }^{+} \mathrm{CD}^{+}{ }^{+}$monocytes was without significant differences in $\mathrm{HV}$ and $\mathrm{TP}$ (HV: $57.3 \%$, data not shown). LAC stimulation of samples obtained from TP showed significantly decreased levels of the TLR4 expression on $\mathrm{HLA}-\mathrm{DR}^{+} \mathrm{CD}_{1}{ }^{+}$monocytes at $\mathrm{ED}$ with no further significant changes in posttraumatic phase compared to stimulated samples from $\mathrm{HV}$ (HV: $74.0 \%$, ED: $49.8 \%, p<$ 0.05 , data not shown).

3.8.3. Surface Expression of TLR9 on HLA-DR ${ }^{-}$and HLA-DR ${ }^{+}$ CD14 ${ }^{+}$Monocytes. $64.5 \%$ of all HLA-DR ${ }^{-} \mathrm{CD}_{14}{ }^{+}$monocytes from $\mathrm{HV}$ expressed TLR9. Starting at day 1, the TLR9 expression was significantly decreased in samples obtained from TP compared to HV for the whole time course $(p<$ 0.05 , Figure $4(\mathrm{f}))$. In HV LAC stimulation increased significantly the TLR9 expression to $88.9 \%$ (Figure $4(\mathrm{f})$ ). The TLR9 expression in HLA-DR ${ }^{-} \mathrm{CD}_{1} 4^{+}$monocytes after LAC stimulation was only significantly lowered in ED samples compared to simulated samples of HV. In the further study period, the value was comparable to stimulated samples of HV (Figure 4(f)).

The TLR9 expression in HLA-DR ${ }^{+} \mathrm{CD}_{14}^{+}$monocytes was significantly decreased at the ED in TP (HV: $32.9 \%$, ED: 20.2\%, $p<0.05$, data not shown); after day 1, the TLR9 expression was comparable to HV. However, the TLR9 expression in stimulated $\mathrm{HLA}-\mathrm{DR}^{+} \mathrm{CD} 4^{+}$monocytes of TP was significantly lower until day 8 after trauma compared to LAC-stimulated samples from HV (data not shown).

TLR2, TLR4, and TLR9 in Coexpression with HLA-DR on $C D 14^{+}$Monocytes. In order to identify the potency of antigenpresenting $\mathrm{CD}_{14}{ }^{+}$monocytes to detect bacterial toxins, we 


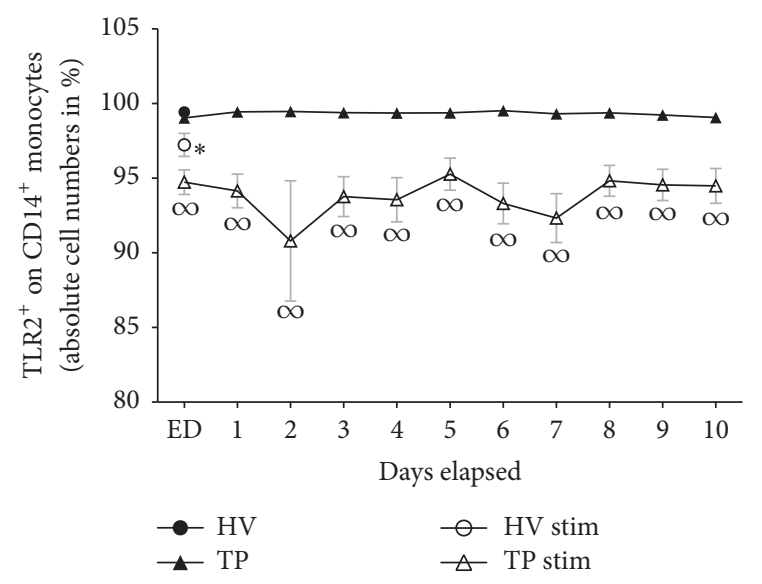

(a)

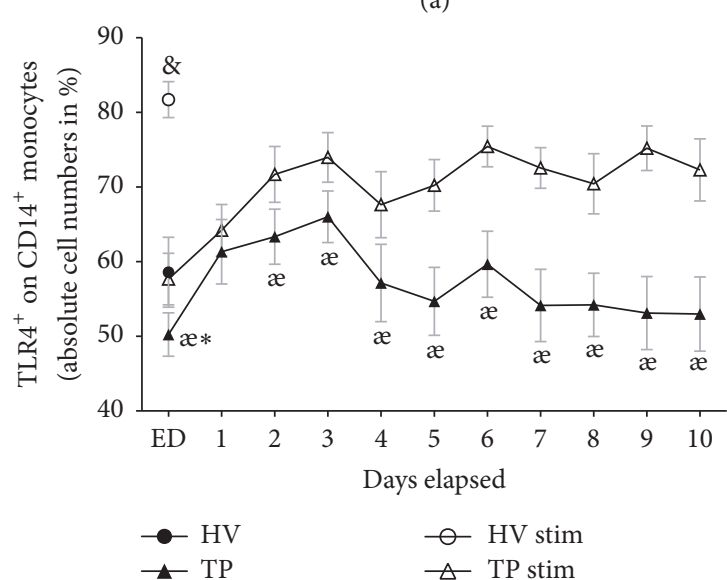

(b)

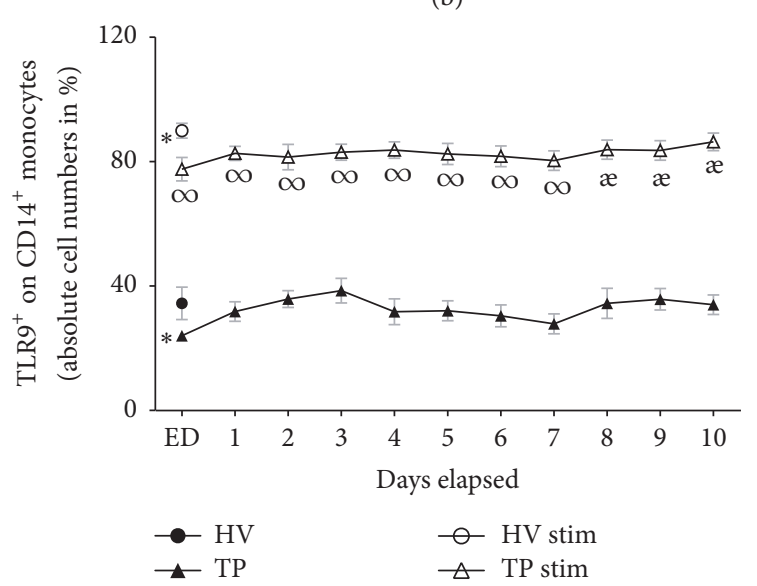

(c)
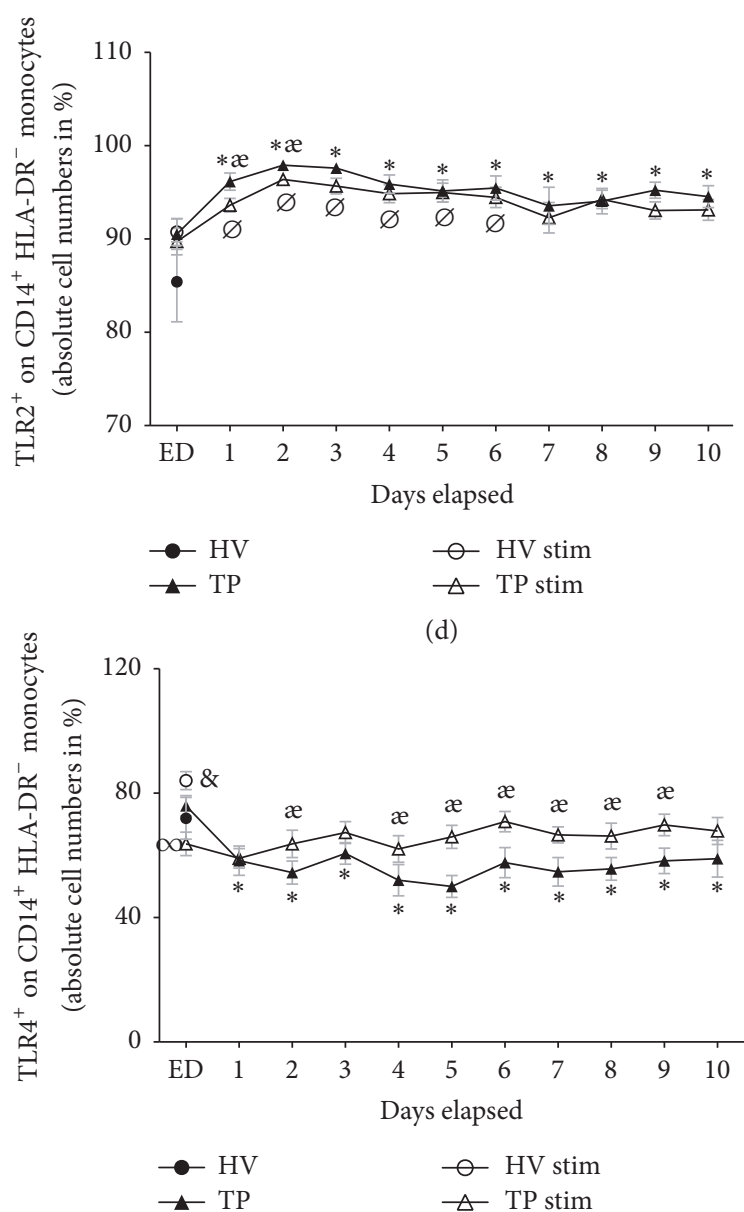

(e)

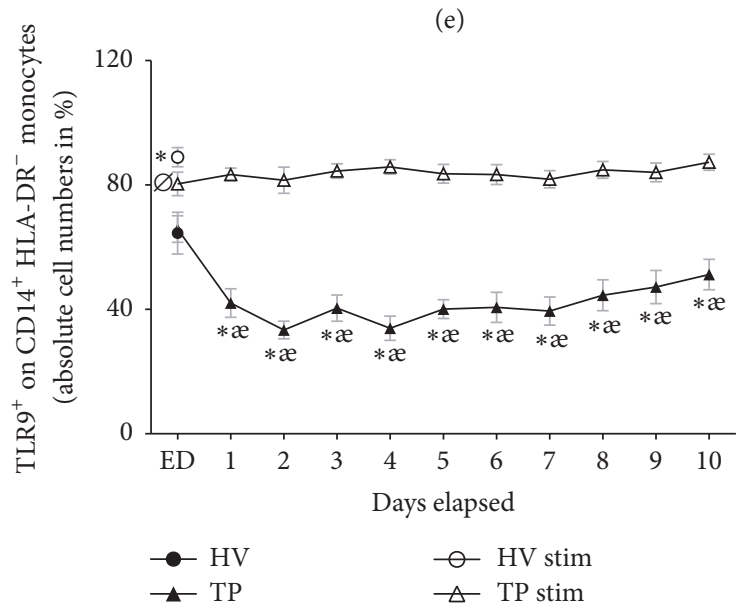

(f)

FIGURE 4: Whole blood from healthy volunteers $(\mathrm{HV}, n=14)$ or major trauma patients (TP, $n=29)$ was analyzed over a 10 -day time course after admission (emergency department, ED-10) by flow cytometry. Monocytes were detected using anti-human CD14 in the corresponding sideward and forward scatter. Unstimulated (black symbols) and stimulated (clear symbols) measurements were made. For stimulation (stim), whole blood was incubated with leukocyte activation cocktail for $5 \mathrm{~h}$ with subsequent analyzing procedure as in unstimulated samples. Data are shown as mean \pm SEM. (a) TLR2 expression in CD14 ${ }^{+}$monocytes, (b) TLR4 expression in CD14 ${ }^{+}$monocytes, (c) TLR9 expression in $\mathrm{CD}_{1}{ }^{+}$monocytes, (d) TLR2 expression in CD14 ${ }^{+}$HLA-DR $^{-}$monocytes, (e) TLR4 expression in CD14 ${ }^{+}$HLA-DR $^{-}$monocytes, and (f) TLR9 expression in $\mathrm{CD}_{14}{ }^{+}$HLA-DR ${ }^{-}$monocytes. ${ }^{*} p<0.05$ versus unstimulated $\mathrm{HV} ;{ }^{\varnothing} p<0.05$ versus stimulated $\mathrm{HV} ;{ }^{\circledR} p<0.05$ TP versus corresponding TP stim; ${ }^{*} p<0.05 \mathrm{HV}$ ctrl versus all; ${ }^{\circledR} p<0.05$ versus all; ${ }^{\infty} p<0.05 \mathrm{TP}$ stim versus corresponding unstimulated TP and HV stim. 


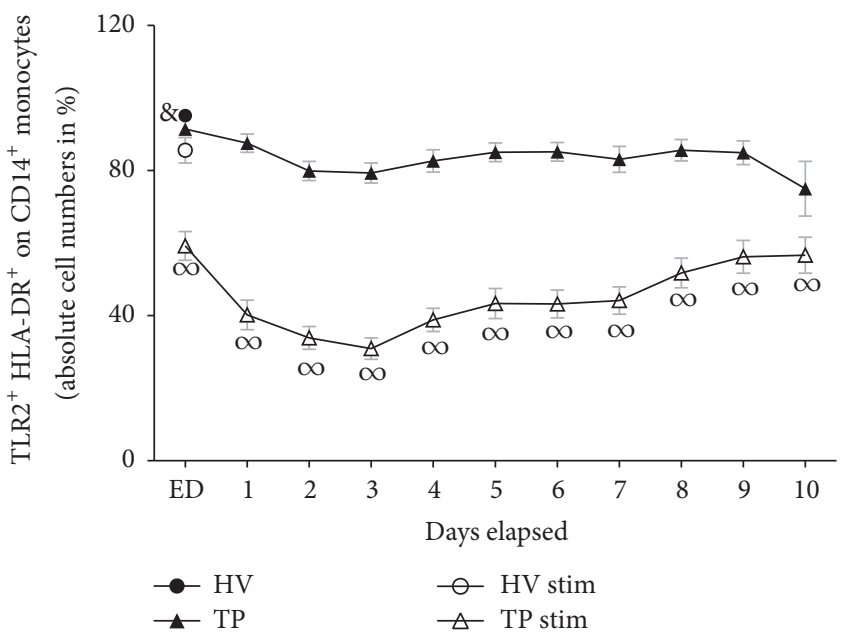

(a)

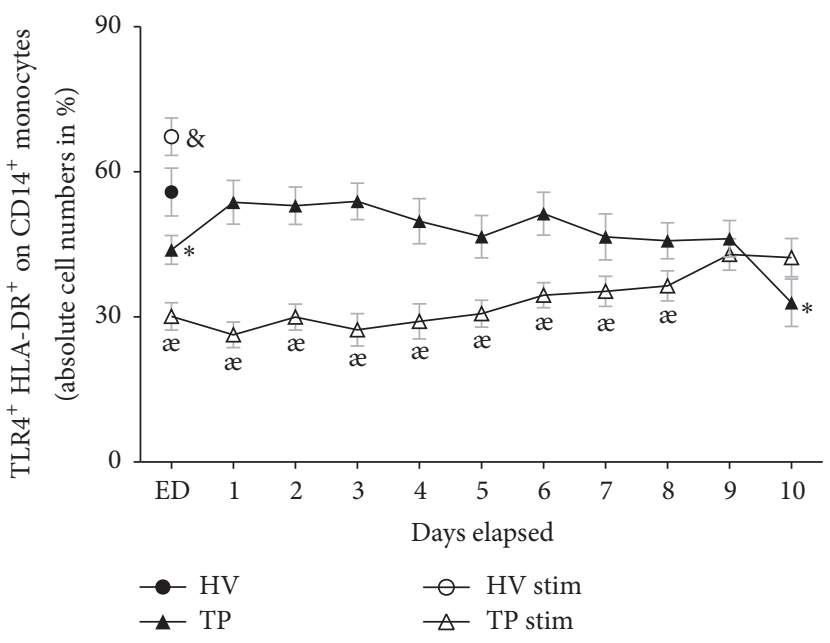

(b)

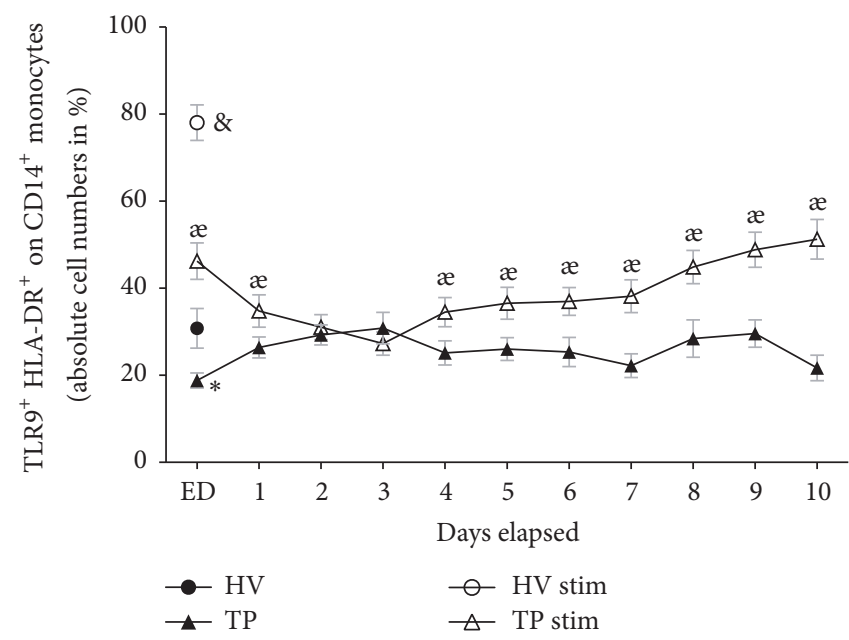

(c)

FIGURE 5: Whole blood from healthy volunteers (HV, $n=14)$ or major trauma patients (TP, $n=29)$ was analyzed by flow cytometry over a 10-day time course after admission (emergency department, ED-10). Monocytes were detected using anti-human CD14 in the corresponding sideward and forward scatter. Unstimulated (black symbols) and stimulated (clear symbols) measurements were made. For stimulation (stim), whole blood was incubated with leukocyte activation cocktail for $5 \mathrm{~h}$ with subsequent analyzing procedure as in unstimulated samples. Data are shown as mean \pm SEM. (a) TLR2/HLA-DR coexpression on CD14 ${ }^{+}$monocytes, (b) TLR4/HLA-DR coexpression on CD14 ${ }^{+}$monocytes, and (c) TLR9/HLA-DR coexpression on CD14 ${ }^{+}$monocytes. ${ }^{*} p<0.05$ versus unstimulated $\mathrm{HV} ;{ }^{\mathfrak{}} p<0.05$ TP versus corresponding TP stim; ${ }^{\&} p<0.05$ versus all; ${ }^{\infty} p<0.05 \mathrm{TP}$ stim versus corresponding unstimulated TP and HV stim.

measured the surface expression of TLR2, TLR4, and TLR9 in coexpression with HLA-DR on mature CD14 ${ }^{+}$monocytes.

During the whole posttraumatic observational period of ten days, the TLR2 and HLA-DR coexpression was significantly reduced in TP compared to $\mathrm{HV}$ (HV: $95.2 \%$ versus ED; $91.4 \pm 1.5, p<0.05$, Figure $5(\mathrm{a})) .55 .8 \%$ of all $\mathrm{CD}^{+} 4^{+}$monocytes from HV coexpressed TLR $4^{+} \mathrm{HLA}^{-\mathrm{DR}^{+}}$and $30.8 \%$ of all $\mathrm{CD} 14^{+}$monocytes from HV coexpressed TLR9 HLA-DR. Both TLR4 HLA-DR coexpression and TLR9 HLA-DR coexpression were significantly reduced in ED. Then, they rose markedly in TP at day 1 compared to samples from ED and reached comparable coexpression levels to $\mathrm{HV}$ in the further posttraumatic course (TLR4 and HLA-DR coexpression ED: 43.9 \pm 2.9 , Figure 5(b); TLR9 and HLA-DR coexpression ED: $18.8 \pm 1.7$ Figure 5(c)). After LAC stimulation, the HLA-DR coexpression of all measured TLRs remained at significantly lower levels in TP during the whole study period compared to stimulated samples from HV (Figures 5(a)-5(c)).

\section{Discussion}

Impaired capability of monocytes to release proinflammatory cytokines upon a secondary ex vivo-in vitro endotoxin exposure, a phenomenon termed as endotoxin tolerance, has been described in several studies for septic and trauma patients $[9,10,36,43,44]$. In line with these findings, we show that stimulating trauma patient's blood samples with LPS diminished the synthesis of IL- $1 \beta$ during the observation period of ten days. Interestingly, a recovery of the IL- $1 \beta$ release upon stimulation began at day 5 . The increased ratio 
of monocytes to leukocytes at postinjury days 6 and 7 may be one possible explanation for the observed IL- $1 \beta$ recovery. Other studies reporting a monocytosis starting at day 5 after trauma confirm our data [13]. After a secondary stimulation with LAC, CD14 expression was comparable in TP and HV. This result indicates that LAC stimulation appears rather specific to PRRs and HLA-DR, but not to CD14.

Severe trauma causes an immune dysfunction with subsequently elevated risk for multiorgan failure and infectious complications $[45,46]$. Monocytes, which play a pivotal role in inflammation, show a lack in phagocytosis [35], decreased HLA-DR expression $[15,16]$, and an impaired cytokine secretion after an ex vivo stimulation with endotoxin in TP $[9,10,36]$. Despite numerous studies in the last decades, the detailed mechanisms of endotoxin tolerance are still not fully described [47-49]. The initial trigger for the production of proinflammatory cytokines is the signal transduction of LPS via TLR4, which appears on the cell surface $[25,50]$. Thus, considering endotoxin tolerance, it seems reasonable to doubt the expression profile of different TLRs on monocytes after trauma. Attempts to correlate the TLR2 and TLR4 expression with the diminished immune activity on monocytes after severe trauma delivered inconsistent results [3437]. However, these studies demonstrated an impaired function of these cells with a reduced proinflammatory cytokine release upon TLR4 stimulation [34-37]. Our data confirm the reduced monocyte activity upon a secondary stimulation after trauma (Figure 2). In fact, these studies differ notably in methods that were applied to describe the TLRs, but they also differed with regard to the timing of acquiring blood samples or the analyzed cohort of patients. Nevertheless, considering these studies, it seems that there is no correlation between the TLR expressions with the diminished cytokine release by monocytes after trauma. It is important to keep in mind that in all these studies the TLR2 or TLR4 expression was evaluated without considering the possible changes in the TLRs (co-)expression after their secondary ex vivo-in vitro stimulation.

The present study showed that the TLR2 expression was not significantly altered in unstimulated blood samples obtained from TP. Surface expression of TLR4 and TLR9 was significantly decreased only at ED (Figures 4(a)-4(c)). In summary, analyzing the native surface expression of TLRs on monocytes from TP did not deliver an explanation for the reduced capability to release IL-1 $\beta$ in monocyte (Figure 2 ). Tsujimoto et al. showed an increase of TLR2 and TLR4 in septic and surgical trauma patients in comparison to a control group. However, the control group expressed higher levels of TLR2 and TLR4 after the ex vivo-in vitro LPS exposure compared to surgical and septic patients [51]. In addition, an upregulation of the TLR9 gene expression in mouse macrophages was shown after LPS stimulation [52]. While the expression levels of TLR4 and TLR9 in our study were higher, TLR2 expression was lower after stimulation. Comparing samples from TP and HV, the capability of expressing TLR2, TLR4, and TLR9 after LAC stimulation was lower in TP (Figures 4(a)-4(c)). The decreased expression of TLR2 after LAC stimulation was profound in TP. A secondary stimulation after trauma may lead to an internalization or an ejection of TLR2 from $\mathrm{CD}_{1}{ }^{+}$monocytes. Diminished TLR2 expression in monocytes from surgical patients after their LPS exposure has been reported previously [51]. Next to the impaired release of IL- $1 \beta$, the impaired capability of expressing TLR4 in ex vivo-in vitro simulated monocytes may be partly responsible for the well-described endotoxin tolerance in trauma patients. Considering the general context, the immunological alterations after trauma appear rather unspecific, and several mechanisms may be involved in endotoxin tolerance via the TLR4 pathway in monocytes [53]. To name only a few, an elevated expression of IL-1 receptor associated kinase-M (IRAK-M) mRNA, a negative regulator of intracellular TLRs signalling cascade [54], has been found in monocytes from septic and endotoxin tolerant patients [55]. Furthermore a downregulation of Nlrp1 inflammasome, which is necessary for IL- $1 \beta$ synthesis, has been described in trauma patients [11].

During the complete observational period, ex vivo-in vitro stimulated $\mathrm{CD}_{14}{ }^{+}$monocytes obtained from TP coexpressed significantly lower levels of $\mathrm{HLA}_{-} \mathrm{DR}^{+} \mathrm{TLR}^{+}{ }^{+}$, HLA$\mathrm{DR}^{+} \mathrm{TLR} 4^{+}$, and HLA-DR ${ }^{+} \mathrm{TLR}{ }^{+}$compared to HV. Thus, the detection of bacterial stimuli after a secondary inflammatory "hit" may be impaired in TP and cause a subsequently delayed activation of further immune cells. The evaluation of unstimulated samples uncovered impaired levels of $\mathrm{HLA}^{-\mathrm{DR}^{+} \mathrm{TLR}^{+}}{ }^{+}$in TP compared to HV during the whole observation period, while the HLA-DR ${ }^{+}$TLR $4^{+}$and HLA$\mathrm{DR}^{+} \mathrm{TLR}^{+}$coexpressions were decreased in unstimulated samples at ED compared to $\mathrm{HV}$.

mHLA-DR may serve as a prognostic marker for trauma and septic patients. Lower levels of HLA-DR on the cell surface have been associated with the development of sepsis after severe trauma $[17,56]$. As expected, the HLA-DR expression on monocytes from TP supported the results of previous reports $[15,36,57]$. TP expressed significantly lower levels of mHLA-DR compared to HV, even more profound after ex vivo-in vitro stimulation (Figure 3(b)). Based on these data, reduced mHLA-DR expression may contribute to the higher susceptibility to infectious or even septic conditions as well. Similar findings reported before by others in septic patients showing depressed mHLA-DR expression support this hypothesis $[58,59]$. The mechanisms that lead to lowered levels of mHLA-DR in septic and trauma patients are not fully understood yet. Possibly, a transcriptional downregulation of genes required for HLA-DR expression may be responsible for its diminished expression in septic patients [58]. Other authors postulated that a partially IL10-mediated reendocytosis of HLA-DR molecules may be responsible for its lower surface expression in patients with septic shock [59]. Another study has demonstrated that the reduced percentage of HLA-DR expression may be explained by increased absolute numbers of CD14 ${ }^{+} \mathrm{HLA}_{-} \mathrm{DR}^{-}$monocytes [60]. $\mathrm{CD}^{+}{ }^{+} \mathrm{HLA}^{-\mathrm{DR}^{-}}$cells have been characterized as a subset of myeloid derived suppressor cells (MDSC), which have immunosuppressive characteristics [61, 62]. After major surgical trauma and in septic patients, CD14 ${ }^{+}$HLA$\mathrm{DR}^{-}$monocytes have been shown to be upregulated as well $[63,64]$. In line with these data, we found increased levels of $\mathrm{CD}^{+} 4^{+} \mathrm{HLA}^{-\mathrm{DR}^{-}}$cells in TP, especially after 
the ex vivo-in vitro stimulation. Elevated levels of $\mathrm{CD} 14^{+}$ HLA-DR ${ }^{-}$cells may partly contribute to endotoxin tolerance after trauma. It is known that severe injury and surgical trauma increase the transmission of haematopoietic progenitor cells from bone marrow $[65,66]$, whereas the function and ability to grow out of bone marrow in culture were suppressed after severe injury [66]. These findings and our own data indicate a lower stage of maturation of monocytes with subsequently impaired function.

There are only sparse data concerning the expression characteristics of TLRs on CD14 ${ }^{+} \mathrm{HLA}_{-} \mathrm{DR}^{-}$cells after trauma. Here, CD14 ${ }^{+}$HLA-DR ${ }^{-}$monocytes expressed less TLR4 and TLR9 in TP compared to HV in native samples. After stimulation, the TLR4 expression on $\mathrm{CD}_{14}{ }^{+} \mathrm{HLA}_{-} \mathrm{DR}{ }^{-}$monocytes was lowered during the whole observational period compared to HV. Due to the observation of an increased ratio of $\mathrm{CD}^{+}{ }^{+} \mathrm{HLA}^{-\mathrm{DR}}{ }^{-}$cells after trauma, this selected subgroup may contribute to the impaired TLR4 expression on stimulated $\mathrm{CD} 14^{+}$monocytes after trauma.

\section{Key Conclusions}

(I) Reduced IL-1 $\beta$ response after ex vivo-in vitro LPS stimulation was paralleled by an impaired TLR4 expression in stimulated monocytes obtained from TP.

(II) In addition, an impaired TLR2 and TLR9 expression in monocytes from TP after their secondary ex vivoin vitro simulation compared to $\mathrm{HV}$ was observed. Unstimulated samples showed significant reduction of TLR4 and TLR9 directly after admission.

(III) HLA-DR expression was lower and even more profound by LAC stimulation of monocytes after trauma.

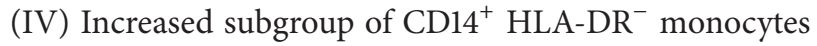
expressed lower levels of TLR4 and TLR9 after trauma. After stimulation, the TLR4 expression was lowered during the whole observational period compared to HV. Due to a possibly limited signal transduction via TLR4 or limited function of monocytes as determined by their lower stage of maturation, these alterations may contribute to the endotoxin tolerance in TP.

(V) The ratio of monocytes to leukocytes was significantly increased at days 6 and 7 after trauma. This modulation may be involved in the observed recovery of the IL- $1 \beta$ release upon LPS stimulation that began at day 5 after trauma.

(VI) Coexpression of different TLRs and HLA-DR on stimulated monocytes from TP was impaired, an effect that may cause a delayed activation of further immune cells after bacterial stimuli.

\section{Abbreviations}

AIS: Abbreviated injury scale

BSA: Bovine serum albumin

\begin{tabular}{|c|c|}
\hline CARS: & $\begin{array}{l}\text { Compensatory anti-inflammatory } \\
\text { response syndrome }\end{array}$ \\
\hline CD: & Cluster of differentiation \\
\hline Ctrl: & Control \\
\hline DAMP: & Damage-associated molecular pattern \\
\hline ED: & Emergency department \\
\hline EDTA: & Ethylenediaminetetraacetic acid \\
\hline ELISA: & Enzyme-linked immunosorbent assay \\
\hline FCS: & Fetal calf serum \\
\hline HLA-DR: & Human leukocyte antigen-DR \\
\hline $\mathrm{HV}:$ & Healthy volunteers \\
\hline ICU: & Intensive care unit \\
\hline IL: & Interleukin \\
\hline IRAK-M: & Interleukin-1 receptor associated kinase- $M$ \\
\hline ISS: & Injury severity score \\
\hline LAC: & Leukocyte activation cocktail \\
\hline LPS: & Lipopolysaccharide \\
\hline MARS: & $\begin{array}{l}\text { Mixed anti-inflammatory response } \\
\text { syndrome }\end{array}$ \\
\hline MDSC: & Myeloid derived suppressor cells \\
\hline MHC-II: & Major histocompatibility complex class II \\
\hline mHLA-DR: & Monocyte human leukocyte antigen-DR \\
\hline MOF: & Multiorgan failures \\
\hline mRNA: & Messenger ribonucleic acid \\
\hline PBS: & Phosphate-buffered saline \\
\hline PICS: & $\begin{array}{l}\text { Persistent inflammation, } \\
\text { immunosuppression, and catabolism } \\
\text { syndrome }\end{array}$ \\
\hline PRR: & Pattern recognition receptors \\
\hline SEM: & Standard error of the mean \\
\hline SIRS: & $\begin{array}{l}\text { Systemic inflammatory response } \\
\text { syndrome }\end{array}$ \\
\hline TLR: & Toll-like receptor \\
\hline TNF-alpha: & Tumor necrosis factor-alpha \\
\hline TP: & Trauma patients. \\
\hline
\end{tabular}

\section{Additional Points}

Short Summary. Reduced LPS response after trauma was paralleled by diminished expression of TLRs and HLA-DR on monocytes compared with healthy volunteers after ex vivo-in vitro stimulation.

\section{Disclosure}

Parts of this study were presented at the XVI. Meeting of the European Shock Society 2015 in Cologne, Germany, as meeting abstracts [67].

\section{Competing Interests}

The authors state that they have no conflict of interests.

\section{Authors' Contributions}

Borna Relja designed the study, obtained the ethical approval, performed the statistical analysis, and revised the manuscript. David Heftrig collected samples, carried out all analysis, and made the first draft of the manuscript. Ramona Sturm 
collected samples and carried out analysis. Elsie Oppermann, Kerstin Kontradowitz, and Katrin Jurida carried out analysis. Ingo Marzi, Lukas Schimunek, and Mathias Woschek revised the manuscript and contributed intellectually to the completion of the study.

\section{Acknowledgments}

The authors thank the student assistants of the Department of Trauma, Hand and Reconstructive Surgery, Goethe University, Frankfurt, for their help in obtaining samples from patients.

\section{References}

[1] G. Regel, P. Lobenhoffer, M. Grotz, H. C. Pape, U. Lehmann, and H. Tscherne, "Treatment results of patients with multiple trauma: an analysis of 3406 cases treated between 1972 and 1991 at a German Level I Trauma Center," Journal of Trauma, vol. 38, no. 1, pp. 70-78, 1995.

[2] R. Pfeifer, I. S. Tarkin, B. Rocos, and H.-C. Pape, "Patterns of mortality and causes of death in polytrauma patients-has anything changed?” Injury, vol. 40, no. 9, pp. 907-911, 2009.

[3] T. M. Osborn, J. K. Tracy, J. R. Dunne, M. Pasquale, and L. M. Napolitano, "Epidemiology of sepsis in patients with traumatic injury," Critical Care Medicine, vol. 32, no. 11, pp. 2234-2240, 2004.

[4] S.-Y. Seong and P. Matzinger, "Hydrophobicity: an ancient damage-associated molecular pattern that initiates innate immune responses," Nature Reviews Immunology, vol. 4, no. 6, pp. 469478, 2004.

[5] R. C. Bone, R. A. Balk, F. B. Cerra et al., "Definitions for sepsis and organ failure and guidelines for the use of innovative therapies in sepsis. The ACCP/SCCM Consensus Conference Committee. American College of Chest Physicians/Society of Critical Care Medicine," Chest, vol. 101, pp. 1644-1655, 1992.

[6] K. R. Kasten, H. S. Goetzman, M. R. Reid et al., "Divergent adaptive and innate immunological responses are observed in humans following blunt trauma," BMC Immunology, vol. 11, article 4, 2010.

[7] R. C. Bone, "Sir Isaac Newton, sepsis, SIRS, and CARS," Critical Care Medicine, vol. 24, no. 7, pp. 1125-1128, 1996.

[8] L. F. Gentile, A. G. Cuenca, P. A. Efron et al., "Persistent inflammation and immunosuppression: a common syndrome and new horizon for surgical intensive care," Journal of Trauma and Acute Care Surgery, vol. 72, no. 6, pp. 1491-1501, 2012.

[9] M. Keel, N. Schregenberger, U. Steckholzer et al., "Endotoxin tolerance after severe injury and its regulatory mechanisms," Journal of Trauma, vol. 41, no. 3, pp. 430-437, 1996.

[10] S. Wutzler, M. Maier, M. Lehnert et al., "Suppression and recovery of LPS-stimulated monocyte activity after trauma is correlated with increasing injury severity: a prospective clinical study," Journal of Trauma, vol. 66, no. 5, pp. 1273-1280, 2009.

[11] B. Relja, J. P. Horstmann, K. Kontradowitz et al., "Nlrp1 inflammasome is downregulated in trauma patients," Journal of Molecular Medicine, vol. 93, no. 12, pp. 1391-1400, 2015.

[12] Z. Spolarics, M. Siddiqi, J. H. Siegel et al., "Depressed interleukin-12-producing activity by monocytes correlates with adverse clinical course and a shift toward Th2-type lymphocyte pattern in severely injured male trauma patients," Critical Care Medicine, vol. 31, no. 6, pp. 1722-1729, 2003.
[13] D. S. Walsh, P. Thavichaigarn, K. Pattanapanyasat et al., "Characterization of circulating monocytes expressing HLA-DR or CD71 and related soluble factors for 2 weeks after severe, nonthermal injury," Journal of Surgical Research, vol. 129, no. 2, pp. 221-230, 2005.

[14] C. Schinkel, R. Sendtner, S. Zimmer, and E. Faist, "Functional analysis of monocyte subsets in surgical sepsis," Journal of Trauma, vol. 44, no. 5, pp. 743-749, 1998.

[15] M. J. Hershman, W. G. Cheadle, S. R. Wellhausen, P. F. Davidson, and H. C. Polk Jr., "Monocyte HLA-DR antigen expression characterizes clinical outcome in the trauma patient," British Journal of Surgery, vol. 77, no. 2, pp. 204-207, 1990.

[16] D. H. Livingston, S. H. Appel, S. R. Wellhausen, G. Sonnenfeld, and H. C. Polk, "Depressed interferon gamma production and monocyte HLA-DR expression after severe injury," Archives of Surgery, vol. 123, no. 11, pp. 1309-1312, 1988.

[17] A. Cheron, B. Floccard, B. Allaouchiche et al., "Lack of recovery in monocyte human leukocyte antigen-DR expression is independently associated with the development of sepsis after major trauma," Critical Care, vol. 14, no. 6, article R208, 2010.

[18] M. Keel and O. Trentz, "Pathophysiology of polytrauma," Injury, vol. 36, no. 6, pp. 691-709, 2005.

[19] J. Kohl, "The role of complement in danger sensing and transmission," Immunologic Research, vol. 34, no. 2, pp. 157-176, 2006.

[20] S. Akira, K. Takeda, and T. Kaisho, "Toll-like receptors: critical proteins linking innate and acquired immunity," Nature Immunology, vol. 2, no. 8, pp. 675-680, 2001.

[21] K. Takeda and S. Akira, "Toll-like receptors," Current Protocols in Immunology, vol. 109, article 14, 2015.

[22] S. Akira and K. Takeda, "Toll-like receptor signalling," Nature Reviews Immunology, vol. 4, no. 7, pp. 499-511, 2004.

[23] E. Lien, T. J. Sellati, A. Yoshimura et al., “Toll-like receptor 2 functions as a pattern recognition receptor for diverse bacterial products," Journal of Biological Chemistry, vol. 274, no. 47, pp. 33419-33425, 1999.

[24] R. Schwandner, R. Dziarski, H. Wesche, M. Rothe, and C. J. Kirschning, "Peptidoglycan- and lipoteichoic acid-induced cell activation is mediated by Toll-like receptor 2," Journal of Biological Chemistry, vol. 274, no. 25, pp. 17406-17409, 1999.

[25] A. Poltorak, X. He, I. Smirnova et al., "Defective LPS signaling in $\mathrm{C} 3 \mathrm{H} / \mathrm{HeJ}$ and $\mathrm{C} 57 \mathrm{BL} / 10 \mathrm{ScCr}$ mice: mutations in Tlr4 gene," Science, vol. 282, no. 5396, pp. 2085-2088, 1998.

[26] E. S. Van Amersfoort, T. J. C. Van Berkel, and J. Kuiper, "Receptors, mediators, and mechanisms involved in bacterial sepsis and septic shock," Clinical Microbiology Reviews, vol. 16, no. 3, pp. 379-414, 2003.

[27] S. D. Wright, R. A. Ramos, P. S. Tobias, R. J. Ulevitch, and J. C. Mathison, "CD14, a receptor for complexes of lipopolysaccharide (LPS) and LPS binding protein," Science, vol. 249, no. 4975, pp. 1431-1433, 1990.

[28] H. Hemmi, O. Takeuchi, T. Kawai et al., "A Toll-like receptor recognizes bacterial DNA," Nature, vol. 408, no. 6813, pp. 740$745,2000$.

[29] J. Tian, A. M. Avalos, S.-Y. Mao et al., "Toll-like receptor 9dependent activation by DNA-containing immune complexes is mediated by HMGB1 and RAGE," Nature Immunology, vol. 8, no. 5, pp. 487-496, 2007.

[30] A. Eaton-Bassiri, S. B. Dillon, M. Cunningham et al., "Toll-like receptor 9 can be expressed at the cell surface of distinct populations of tonsils and human peripheral blood mononuclear cells," Infection and Immunity, vol. 72, no. 12, pp. 7202-7211, 2004. 
[31] J. B. Ewaschuk, J. L. Backer, T. A. Churchill, F. Obermeier, D. O. Krause, and K. L. Madsen, "Surface expression of tolllike receptor 9 is upregulated on intestinal epithelial cells in response to pathogenic bacterial DNA," Infection and Immunity, vol. 75, no. 5, pp. 2572-2579, 2007.

[32] K. U. Saikh, T. L. Kissner, A. Sultana, G. Ruthel, and R. G. Ulrich, "Human monocytes infected with Yersinia pestis express cell surface TLR9 and differentiate into dendritic cells," Journal of Immunology, vol. 173, no. 12, pp. 7426-7434, 2004.

[33] E. Juarez, C. Nuñez, E. Sada, J. J. Ellner, S. K. Schwander, and M. Torres, "Differential expression of Toll-like receptors on human alveolar macrophages and autologous peripheral monocytes," Respiratory Research, vol. 11, article no. 2, 2010.

[34] M. Adib-Conquy, P. Moine, K. Asehnoune et al., "Toll-like receptor-mediated tumor necrosis factor and interleukin-10 production differ during systemic inflammation," American Journal of Respiratory and Critical Care Medicine, vol. 168, no. 2, pp. 158-164, 2003.

[35] J. Pérez-Bárcena, V. Regueiro, C. Crespí et al., "Expression of toll-like receptors 2 and 4 is upregulated during hospital admission in traumatic patients: lack of correlation with blunted innate immune responses," Annals of Surgery, vol. 251, no. 3, pp. 521-527, 2010.

[36] S. Lendemans, E. Kreuzfelder, M. Rani et al., “Toll-like receptor 2 and 4 expression after severe injury is not involved in the dysregulation of the innate immune system," Journal of Trauma, vol. 63 , no. 4, pp. 740-746, 2007.

[37] K. Laudanski, A. De, S. Brouxhon, S. Kyrkanides, and C. MillerGraziano, "Abnormal PGE2 regulation of monocyte TNF- $\alpha$ levels in trauma patients parallels development of a more macrophage-like phenotype," Shock, vol. 22, no. 3, pp. 204-212, 2004.

[38] E. von Elm, D. G. Altman, M. Egger, S. J. Pocock, P. C. Gøtzsche, and J. P. Vandenbroucke, "The strengthening the reporting of observational studies in epidemiology (STROBE) statement: guidelines for reporting observational studies," Journal of Clinical Epidemiology, vol. 61, no. 4, pp. 344-349, 2008.

[39] "Rating the severity of tissue damage. I. The abbreviated scale," The Journal of the American Medical Association, vol. 215, no. 2, pp. 277-280, 1971.

[40] AAAM, Abbreviated Injury Scale 2005 update 2008, Association for the Advancement of Automotive Medicine, Barrington, Ill, USA, 2005.

[41] M. Voth, S. Holzberger, B. Auner, D. Henrich, I. Marzi, and B. Relja, "I-FABP and L-FABP are early markers for abdominal injury with limited prognostic value for secondary organ failures in the post-traumatic course," Clinical Chemistry and Laboratory Medicine, vol. 53, no. 5, pp. 771-780, 2015.

[42] B. Relja, T. Lustenberger, B. Puttkammer et al., "Thrombinactivatable fibrinolysis inhibitor (TAFI) is enhanced in major trauma patients without infectious complications," Immunobiology, vol. 218, no. 4, pp. 470-476, 2013.

[43] S. E. Greisman, E. J. Young, and F. A. Carozza Jr., "Mechanisms of endotoxin tolerance. V. Specificity of the early and late phases of pyrogenic tolerance," Journal of Immunology, vol. 103, no. 6, pp. 1223-1236, 1969.

[44] W. Ertel, J.-P. Kremer, J. Kenney et al., "Downregulation of proinflammatory cytokine release in whole blood from septic patients," Blood, vol. 85, no. 5, pp. 1341-1347, 1995.

[45] A. Wafaisade, R. Lefering, B. Bouillon et al., "Epidemiology and risk factors of sepsis after multiple trauma: an analysis of 29,829 patients from the Trauma Registry of the German Society for Trauma Surgery," Critical Care Medicine, vol. 39, no. 4, pp. 621$628,2011$.

[46] S. Wutzler, T. Lustenberger, B. Relja, M. Lehnert, and I. Marzi, "Pathophysiology of multiple trauma: intensive care medicine and timing of treatment," Chirurg, vol. 84, no. 9, pp. 753-758, 2013.

[47] M. Lacatus, "Innate immunity in surgical patients," Chirurgia (Romania), vol. 108, no. 1, pp. 18-25, 2013.

[48] E. López-Collazo and C. del Fresno, "Pathophysiology of endotoxin tolerance: mechanisms and clinical consequences," Critical Care, vol. 17, no. 6, article no. 242, 2013.

[49] M. Morris and L. Li, "Molecular mechanisms and pathological consequences of endotoxin tolerance and priming," Archivum Immunologiae et Therapiae Experimentalis, vol. 60, no. 1, pp.1318, 2012.

[50] B. Beutler, X. Du, and A. Poltorak, "Identification of toll-like receptor 4 (Tlr4) as the sole conduit for LPS signal transduction: genetic and evolutionary studies," Journal of Endotoxin Research, vol. 7, no. 4, pp. 277-280, 2001.

[51] H. Tsujimoto, S. Ono, T. Majima et al., "Differential toll-like receptor expression after ex vivo lipopolysaccharide exposure in patients with sepsis and following surgical stress," Clinical Immunology, vol. 119, no. 2, pp. 180-187, 2006.

[52] H. An, H. Xu, Y. Yu et al., "Up-regulation of TLR9 gene expression by LPS in mouse macrophages via activation of NF- $\kappa \mathrm{B}$, ERK and p38 MAPK signal pathways," Immunology Letters, vol. 81, no. 3, pp. 165-169, 2002.

[53] S. K. Biswas and E. Lopez-Collazo, "Endotoxin tolerance: new mechanisms, molecules and clinical significance," Trends in Immunology, vol. 30, no. 10, pp. 475-487, 2009.

[54] K. Kobayashi, L. D. Hernandez, J. E. Galán, C. A. Janeway Jr., R. Medzhitov, and R. A. Flavell, "IRAK-M is a negative regulator of Toll-like receptor signaling," Cell, vol. 110, no. 2, pp. 191-202, 2002.

[55] P. Escoll, C. Del Fresno, L. García et al., "Rapid up-regulation of IRAK-M expression following a second endotoxin challenge in human monocytes and in monocytes isolated from septic patients," Biochemical and Biophysical Research Communications, vol. 311, no. 2, pp. 465-472, 2003.

[56] M. Ditschkowski, E. Kreuzfelder, V. Rebmann et al., "HLA-DR expression and soluble HLA-DR levels in septic patients after trauma," Annals of Surgery, vol. 229, no. 2, pp. 246-254, 1999.

[57] C.-X. Hu, X. Xu, H.-P. Liang, F.-L. Lu, L.-Q. Shen, and T.-Z. Hao, "Changes in human leukocyte antigen-DR expression on monocytes and its value of prediction on infection complication in trauma patients," Chinese Critical Care Medicine, vol. 16, no. 4, pp. 193-197, 2004.

[58] A. Pachot, G. Monneret, A. Brion et al., "Messenger RNA expression of major histocompatibility complex class II genes in whole blood from septic shock patients," Critical Care Medicine, vol. 33, no. 1, pp. 31-38, 2005.

[59] T. Fumeaux and J. Pugin, "Role of interleukin-10 in the intracellular sequestration of human leukocyte antigen-DR in monocytes during septic shock," American Journal of Respiratory and Critical Care Medicine, vol. 166, no. 11, pp. 1475-1482, 2002.

[60] F. Hietbrink, L. Koenderman, M. Althuizen, J. Pillay, V. Kamp, and L. P. H. Leenen, "Kinetics of the innate immune response after trauma: implications for the development of late onset sepsis," Shock, vol. 40, no. 1, pp. 21-27, 2013. 
[61] B. Hoechst, L. A. Ormandy, M. Ballmaier et al., "A new population of myeloid-derived suppressor cells in hepatocellular carcinoma patients induces CD4(+)CD25(+)Foxp3(+) T cells," Gastroenterology, vol. 135, pp. 234-243, 2008.

[62] P. Filipazzi, R. Valenti, V. Huber et al., "Identification of a new subset of myeloid suppressor cells in peripheral blood of melanoma patients with modulation by a granulocyte-macrophage colony-stimulation factor-based antitumor vaccine," Journal of Clinical Oncology, vol. 25, no. 18, pp. 2546-2553, 2007.

[63] M. Albertsmeier, D. Quaiser, V. Von Dossow-Hanfstingl, H. Winter, E. Faist, and M. K. Angele, "Major surgical trauma differentially affects T-cells and APC, Innate Immunity, vol. 21, no. 1, pp. 55-64, 2015.

[64] H. Janols, C. Bergenfelz, R. Allaoui et al., "A high frequency of MDSCs in sepsis patients, with the granulocytic subtype dominating in gram-positive cases," Journal of Leukocyte Biology, vol. 96, no. 5, pp. 685-693, 2014.

[65] I. Grzelak, W. L. Olszewski, M. Zaleska et al., "Surgical trauma evokes a rise in the frequency of hematopoietic progenitor cells and cytokine levels in blood circulation," European Surgical Research, vol. 30, no. 3, pp. 198-204, 1998.

[66] D. H. Livingston, D. Anjaria, J. Wu et al., "Bone marrow failure following severe injury in humans," Annals of Surgery, vol. 238, no. 5, pp. 748-753, 2003.

[67] D. Heftrig, R. Sturm, I. Marzi, and B. Relja, "Impaired TLR4 expression on either stimulated or naive CD14+ monocytes may be responsible for their functional suppression after trauma," in Proceedings of the 16th Meeting of the European Shock Society, Cologne, Germany, 2015. 


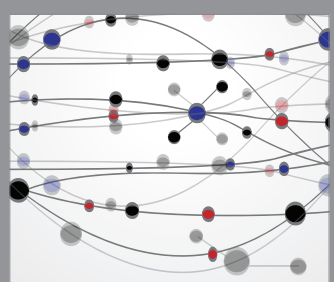

The Scientific World Journal
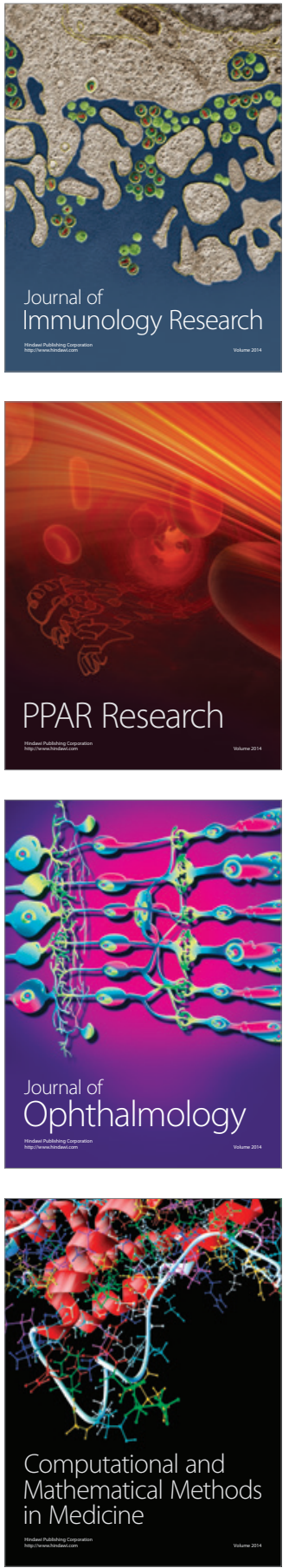

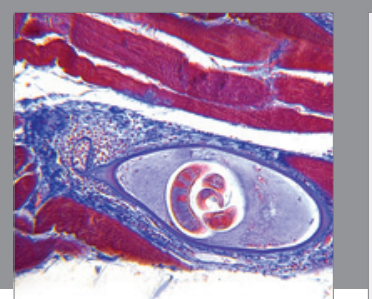

Gastroenterology Research and Practice
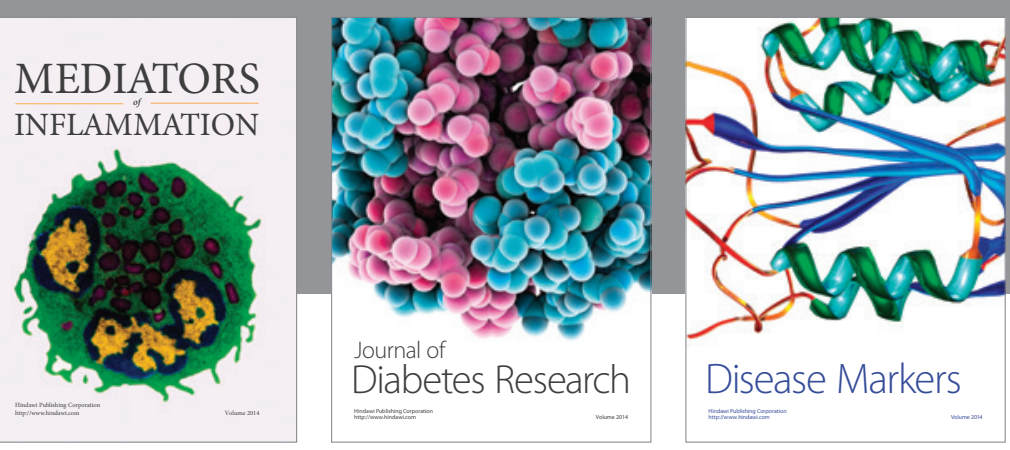

Disease Markers

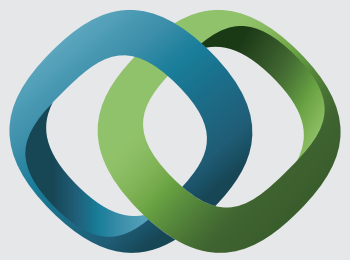

\section{Hindawi}

Submit your manuscripts at

https://www.hindawi.com
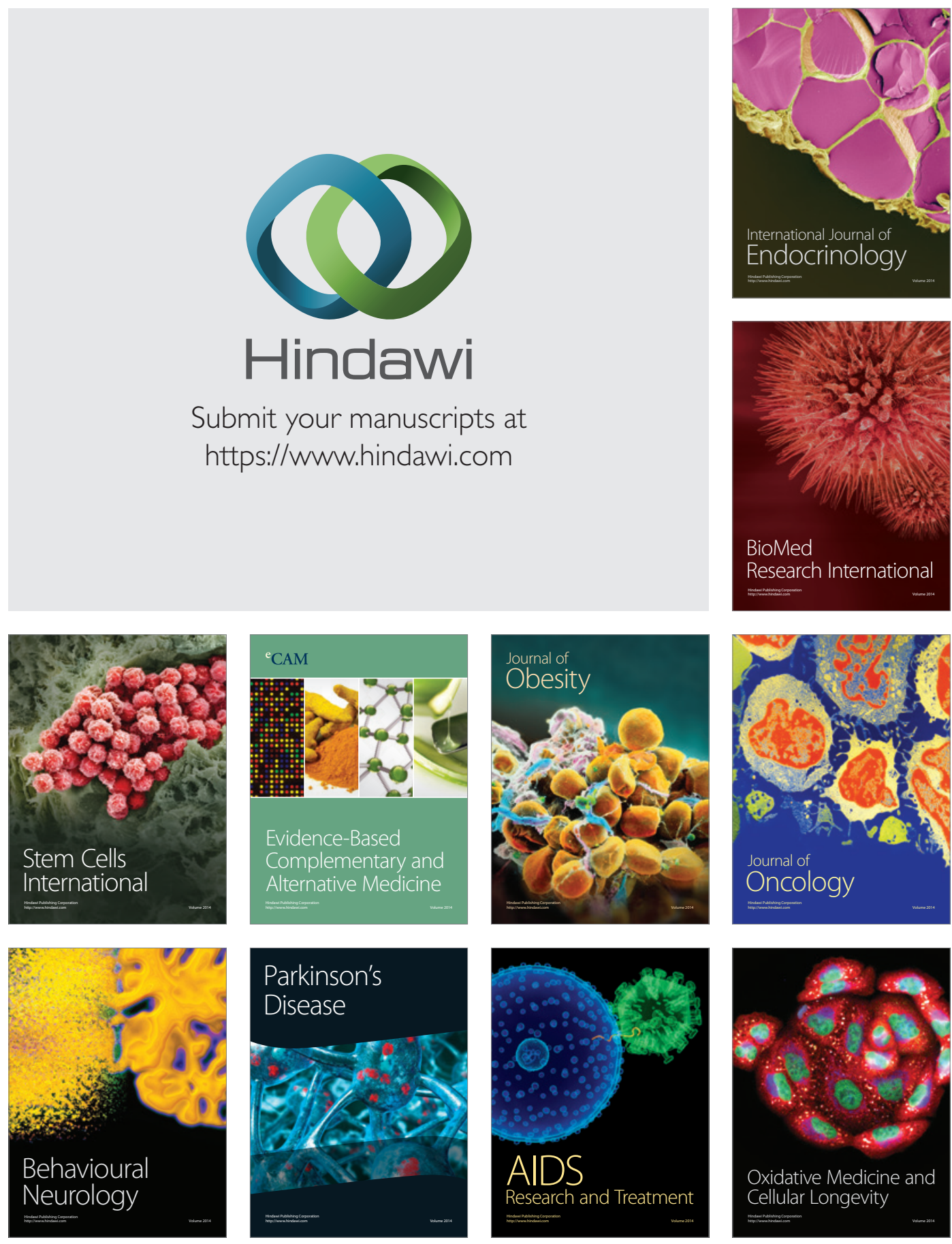